\title{
Opioides y adicción en dolor crónico: ¿es un problema?, ¿cómo abordarlo?
}

El uso de opioides en el dolor crónico no oncológico sufrió un incremento importante en el mundo en los últimos 20 años y en España, aunque más tardíamente, ha existido también un ascenso muy importante en los últimos años, si bien es verdad que partíamos de unas dosis muy bajas de prescripción de opioides y que este incremento se ha producido fundamentalmente a expensas de dosis bajas de tramadol. Sin embargo, paralelamente también se ha producido un uso elevado del fentanilo, una parte no despreciable en las formulaciones de liberación rápida [1].

Cualquier profesional sanitario es consciente del problema desencadenado en EE. UU. por el consumo aberrante de opioides y las consecuencias negativas que ha tenido en la salud de los pacientes y el impacto en las políticas sanitarias en este país [2].

Dice un refrán castellano que cuando las barbas de tu vecino veas pelar, pongas las tuyas a remojar. Y en este sentido podríamos preguntarnos si en Europa, y más concretamente en España, este incremento en el uso de opioides se ve acompañado de un incremento del riesgo de adicción a los mismos y podría desencadenar efectos tan deletéreos como en EE. UU., donde por primera vez la esperanza de vida en la población americana ha disminuido debido a este problema. Pues claramente, ni en Europa ni en España el consumo de opioides se ha visto acompañado de un problema de adicción de forma significativa, sin negar la evidencia de la existencia de la misma en algunos pacientes $[3,4]$.

En este número de la Revista de la Sociedad Española del Dolor se presenta un interesante artículo enviado por los autores Pujante y cols., sobre el uso y mal uso de los fármacos opioides para el tratamiento del dolor, desconexión rápida de opioides-DRAO. En el mismo se pone de relevancia la importancia de problema por la dificultad de diagnóstico. Probablemente los circuitos de la adicción en los pacientes con dolor sean diferentes de los pacientes sin dolor, y el impulso de consumo sea más la evitación de un estado de disforia/depresión que la búsqueda de la euforia y el disfrute. Siendo, por tanto, difícil la aplicación de los métodos de diagnóstico, que realmente han sido pensados para pacientes sin dolor. Por tanto, como bien dicen los autores, necesitamos estar alerta ante los pacientes, especialmente en aquellos que utilizan opioides de liberación ultrarrápida y fuera del entorno de la indicación clínica aprobada (5). Para lograr evitar los peligros potenciales y graves de la adicción a los opioides son importantes: primero una prescripción responsable y justificada, segundo la búsqueda de factores de riesgo, y por último el seguimiento estrecho de los pacientes (6).

A pesar de todos estos datos, la prevalencia es desconocida, no parece ser alarmante en estos momentos (7); de hecho en este artículo de una población de 220.000 habitantes detectan 6 casos. Es posible que parte de esta cifra tan baja sea debido a la falta de diagnóstico y sospecha, excepto en pacientes con un problema muy grave, pero claramente no se acerca a algunas estadísticas de EE. UU., donde se cifra el problema de la adicción en porcentajes superiores al $40 \%$ [8].

Los autores inciden en la importancia del problema. A pesar de su baja prevalencia, al hecho de las devastadoras consecuencias en la vida de un paciente, ya complejo por tener un dolor crónico intenso, se añade otra enfermedad crónica y un elemento de potencial iatrogenia como es la adicción a opioides prescritos. Por lo tanto, y pese a la supuesta baja prevalencia en el momento actual, se hacen necesarias políticas de prevención y también de abordaje clínico en estos pacientes, siendo prioritario la deshabituación de los mismos. Existen muchas dificultades en este sentido que abarcan desde el reconocimiento del 
paciente (9) y su consentimiento para la deshabituación junto al seguimiento posterior por un equipo que incluya psicólogos/psiquiatras expertos en adicciones $(10,11)$.

Desde las Unidades del Dolor es imprescindible crear protocolos de abordaje de estos pacientes, aunque, como relatan los autores, no existe una guía de actuación clara. Uno de los problemas es que muchos de los artículos que proponen desintoxicaciones son muy antiguos; y es cierto que en la actualidad el conocimiento de los mecanismo de acción de los fármacos y el desarrollo en pacientes con altas dosis de opioides de hiperalgesia añadida a la adicción hace muy útil y necesario la valoración de nuevos fármacos en la desconexión rápida de opioides como la ketamina, y como el propofol, asociados o no a otros como la clonidina, o más recientemente la dexmedetomidina (12-17). Como el artículo pone de relevancia, no tenemos un modelo único o con evidencia en estos pacientes y, aunque es preferible un abordaje rápido y eficaz, no debemos renunciar a la seguridad del paciente, siendo fundamental buscar un protocolo que sin ser excesivamente agresivo nos permita una solución rápida inicial, aunque lógicamente esto en ningún momento evita el seguimiento estrecho posterior.

Por lo tanto, estamos ante un problema grave, poco frecuente en el momento actual en nuestro entorno, que aunque precisa de un cambio en nuestras prácticas clínicas que abarque desde la prevención hasta la incorporación de protocolos de tratamiento de la adicción a opioides.

C. Pérez Hernández Jefa de Unidad del Dolor. Hospital Universitario de La Princesa. Madrid. Universidad Alfonso X el Sabio (UAX). Madrid. España

Correspondencia: Concepción Pérez Hernández concha.phte@gmail.com

\section{BIBLIOGRAFÍA}

1. https://www.aemps.gob.es/medicamentosUsoHumano/observatorio/informes-publicados/informesopioides-espana-2010-2017.htm

2. Jones MR, Viswanath O, Peck J, Kaye AD, Gill JS, Simopoulos TT. A Brief History of the Opioid Epidemic and Strategies for Pain Medicine. Pain Ther 2018;7(1):13-21. D0I: 10.1007/s40122-018-0097-6.

3. Häuser W, Petzke F, Radbruch L, Tölle TR. The opioid epidemic and the long-term opioid therapy for chronic noncancer pain revisited: a transatlantic perspective. Pain Manag 2016;6(3):249-63. DOI: 10.2217/pmt.16.5.

4. O'Brien T, Christrup LL, Drewes AM, Fallon MT, Kress HG, McQuay HJ, et al. European Pain Federation position paper on appropriate opioid use in chronic pain management. Eur J Pain 2017;21(1):3-19. DOI: 10.1002/ejp.970.

5. Ballantyne JC. Opioids for the Treatment of Chronic Pain: Mistakes Made, Lessons Learned, and Future Directions. Anesth Analg 2017;125(5):1769-78. DOI: 10.1213/ANE.0000000000002500.

6. Webster LR. Risk Factors for Opioid-Use Disorder and Overdose. Anesth Analg 2017;125(5):1741-8. DOI: 10.1213/ANE.0000000000002496.

7. Higgins C, Smith BH, Matthews K. Incidence of iatrogenic opioid dependence or abuse in patients with pain who were exposed to opioid analgesic therapy: a systematic review and meta-analysis. $\mathrm{Br} \mathrm{J}$ Anaesth 2018;120(6):1335-44. DOl: 10.1016/j.bja.2018.03.009.

8. Cheatle MD. Prescription Opioid Misuse, Abuse, Morbidity, and Mortality: Balancing Effective Pain Management and Safety. Pain Med. 2015;16(Suppl 1):S3-8. DOI: 10.1111/pme.12904.

9. Matthias MS, Krebs EE, Collins LA, Bergman AA, Coffing J, Bair MJ. "I'm not abusing or anything": patientphysician communication about opioid treatment in chronic pain. Patient Educ Couns 2013;93(2):197202. DOI: 10.1016/j.pec.2013.06.021.

10. Manchikanti L, Kaye AM, Knezevic NN, McAnally H, Slavin K, Trescot AM, et al. Responsible, Safe, and Effective Prescription of Opioids for Chronic Non-Cancer Pain: American Society of Interventional Pain Physicians (ASIPP) Guidelines. Pain Physician 2017;20(2S):S3-S92.

11. Kaye AD, Jones MR, Kaye AM, Ripoll JG, Galan V, Beakley BD, et al. Prescription Opioid Abuse in Chronic Pain: An Updated Review of Opioid Abuse Predictors and Strategies to Curb Opioid Abuse: Part 1. Pain Physician 2017;20(2S):S93-S109.

12. Scofield MD, Heinsbroek JA, Gipson CD, Kupchik YM, Spencer S, Smith AC, et al. The Nucleus Accumbens: Mechanisms of Addiction across Drug Classes Reflect the Importance of Glutamate Homeostasis. Pharmacol Rev 2016;68(3):816-71. DOl: 10.1124/pr.116.012484. 
13. Yang DZ, Sin B, Beckhusen J, Xia D, Khaimova R, lliev I. Opioid-Induced Hyperalgesia in the Nonsurgical Setting: A Systematic Review. Am J Ther 2018. DOI: 10.1097/MJT.0000000000000734 [Epub ahead of print].

14. Silverman SM. Opioid induced hyperalgesia: clinical implications for the pain practitioner. Pain Physician 2009ay; 12(3):679-84.

15. lacobucci GJ, Visnjevac O, Pourafkari L, Nader ND. Ketamine: An Update on Cellular and Subcellular Mechanisms with Implications for Clinical Practice. Pain Physician. 2017;20(2):E285-E301.

16. Strickler EM, Schwenk ES, Cohen MJ, Viscusi ER. Use of Ketamine in a Multimodal Analgesia Setting for Rapid Opioid Tapering in a Profoundly Opioid-Tolerant Patient: A Case Report. A A Pract 2018;10(7):17981. DOI: 10.1213/XAA.0000000000000653.

17. Kaye AD, Chung KS, Vadivelu N, Cantemir C, Urman RD, Manchikanti L. Opioid induced hyperalgesia altered with propofol infusion. Pain Physician 2014;17(2):E225-8. 